\title{
Reported knowledge, attitudes and practices regarding malaria and mosquito net use among women seeking antenatal care in south-western Tanzania
}

\author{
EMMANUELA E. AMBROSE ${ }^{1}$, HUMPHREY D. MAZIGO ${ }^{1 *}$, JORG HEUKELBACH ${ }^{3}$, OSCAR \\ GABONE²and DAMAS L. MWIZAMHOLYA ${ }^{4}$ \\ ${ }^{1}$ Weill-Bugando University College of Health Sciences, P.O. Box 1464, Mwanza, Tanzania \\ ${ }^{2}$ Medical Doctor In-charge, Iringa Regional Hospital, P.O. Box Iringa, Tanzania \\ ${ }^{3}$ Department of Community Health, School of Medicine, Federal University of Ceará, Fortaleza, Brazil \\ ${ }^{4}$ Department of Pediatrics, Bugando Medical Centre, P.O. Box 1370, Mwanza, Tanzania
}

\begin{abstract}
To improve control measures against malaria, Tanzania has increased the distribution of free and subsidized insecticide-treated mosquito nets (ITNs) to pregnant women. However, data on ownership and use of ITNs, as well as knowledge, attitudes and practices of these women regarding malaria are scarce. The objective of this study was to describe knowledge, attitudes and practices towards malaria, frequency of bed net use and level of ownership of bed nets among women seeking antenatal care in Iringa regional hospital, southern Tanzania. This cross-sectional study involved women attending antenatal clinic at Iringa Regional Hospital in south-western Tanzania. A pre-tested structured questionnaire was applied. Among the 222 pregnant women included, 173 (78\%, 95\%CI, 72-84.2) owned a bed net, and 150 (68\%, 95\%CI, 61$75)$ reported to sleep always under a bed net. The use of bed nets was mentioned by $142(64 \%$, $95 \% \mathrm{CI}, 56.2-72)$. Of the 46 women who did not own a bed net, seven (15.2\%) reported cost as the main obstacle for owning one. About $53 \%(95 \% \mathrm{CI}, 44-62)$ preferred to use mosquito nets they bought rather than the one provided for free. Several factors such as gravidity, fearing of getting malaria, knowledge on the cause, marital status, and ways used to prevent malaria were significantly associated with mosquito net ownership (all $P<0.001$ ). Education level and gravidity were associated with the behaviour to sleep always under bed nets $(P<0.002)$. Multigravidae $(2-4$ pregnancies) (OR 2.1, 95\%CI 1.2-4.8) and married women (OR, 1.9, 95\%CI, 1.2-5.2) were more likely to own a net, as compared to primigravidae and single women. In conclusion, ITNs ownership and use among pregnant women was good and they preferred to use the nets they bought from private sector. Thus, integrating public and private sector will improve the distribution and coverage of insecticides treated mosquito among high risk groups, such as pregnant women.
\end{abstract}

Keywords: pregnant women, mosquito net, ownership, use, malaria, Tanzania

\section{Introduction}

There are between 300 and 500 million malaria infections and 1 million malaria attributed deaths worldwide each year (Roll Back Malaria/WHO, 2002). About $90 \%$ of these deaths occur in sub-Saharan Africa (Roll Back Malaria/WHO, 2002), and the majority of these cases occur among women and children (Roll Back Malaria/WHO,

\footnotetext{
*Correspondence: Dr. Humphrey D. Mazigo; E-mail: humphreymazigo@gmail.com 
2002). Malaria also presents major obstacles to social and economic development of affected countries. In African countries with stable malaria transmission, infection during pregnancy is estimated to cause about 10,000 maternal deaths each year, $8-14 \%$ of low birth weight babies and 3-8\% of all infant deaths (WHO/UNICEF, 2003). Because of this, pregnant mothers and chhildren under 5 year-olds have been the key targets for malaria prevention, using Insecticides Treated Mosquito Nets (ITNs).

ITNs reduce human contact with infected mosquitoes and have been shown to be an effective malaria prevention measure (Roll Back Malaria, 2005). Use of ITNs among pregnant women is associated with lower prevalence of malaria infections, lower occurrence of premature birth and significant reductions maternal anaemia (D'Alessandro et al., 2003; ter Kuile et al., 2003). In addition to the direct benefit to the individual, ITNs use offers a protective benefit for the entire community. Increased ITNs use throughout a community reduces transmission and thus has a more powerful impact than individual change (Teklehaimonot et al., 2007).

In 2004, the National Malaria Control Programme (NMCP) introduced a voucher subsidy for pregnant women as part of a nation-wide programme to prevent malaria by enhancing coverage of pregnant women and the young children who share their sleeping spaces during and after the pregnancy. Although distribution of ITNs to pregnant mothers and under 5 year-olds has been massively expanded in Tanzania, there is little information on the frequency of ownership, knowledge, attitudes and practices (KAP) of pregnant women on ITNs use. Some studies have suggested that mosquitoes nuisance, malaria knowledge and socio-demographic factors, including education and household income are important determinants of bed net ownership and use (Opiyo et al., 2007, Wiseman et al., 2007, Mazigo et al., 2010). However, knowledge on the benefits of ITNs or ownership of ITNs does not necessarily imply use. Therefore, the objective of this study was to investigate the level of bed net ownership and use, as well as KAP towards malaria and ITNs use, among pregnant women attending an antenatal care clinic. Understanding the factors involved with bed net ownership and use in pregnant women is essential for refining ITNs distribution and for developing effective information, education and behavioral change activities.

\section{Material and Methods}

\section{Study area, design and population}

This cross-sectional study was carried out between July and September 2009 at Iringa Regional Hospital. Iringa municipality is located between latitude $7^{\circ} 49^{\prime}$ south to longitude $35^{\circ} 15^{\prime}$ east and lies at $1560-2000 \mathrm{~m}$ altitude. The area receives an annual rainfall of $750-1000 \mathrm{~mm}$, mostly between December and April. The mean annual temperature is about $19^{\circ} \mathrm{C}$.

The study focused on women attending antenatal clinics (ANC) at the hospital. Pregnant women who were at least 14 years old and consented to be interviewed were eligible to participate in the study. Participants were recruited consecutively until the sample size of 222 pregnant women was reached. Study participants were interviewed 
at the ANC in Kiswahili language by the principal investigator (EEA), using a pre-tested structured questionnaire. The questionnaire covered socio-demographic information, questions on bed net ownership, information about bed net use, as well as questions on knowledge, attitudes and practices about malaria, bed net use and malaria control activities. To guarantee privacy of the information from the participants, pregnant women were interviewed individually by the investigator in a room which was allocated at ANC. The interview was conducted after pregnant women had received all the ANC services.

\section{Data management and analysis}

Data entry was done in Microsoft Access (Microsoft Corp., Redmond, USA) in duplicate, and datasets were checked for entry-related errors. Data were analyzed using SPSS version 11.5 for Window (SPSS Inc, Chicago, IL, USA). Frequency tables were generated and cross tabulations of important variables done. Bivariate analyses were conducted to determine factors associated with net ownership and net usage using chi-squared statistics.

\section{Ethical considerations}

The study was carried out as part of an elective course of the principal investigator (Emmanuela E. Ambrose) and received ethical clearance from Iringa regional hospital management and Weill-Bugando University College of Health Sciences. General information regarding the nature of the study and its objectives was explained to participants. Confidentiality and anonymity was maintained by using codes instead of participants' names. Inclusion of participants took place after informed written consent was received.

\section{Results}

\section{Socio-demographic characteristics of the study population}

A total of 222 pregnant women consented to be interviewed and were included in data analysis. Table 1 details the demographic characteristics of the study population. The mean age was 29.5 years ( $\mathrm{SD}=7.2$ years) and ranged between 14 and 45 years. Most of the women were married $(71.6 \%)$, while seven (3.2\%) were single living alone, and another $43(19.5 \%)$ living with their parents. The majority of women $(54.1 \%)$ were in their second to fourth pregnancy, and few were gradimultipara. About 50\% of the respondents had completed primary school education, while few had secondary education.

Table 1: Demographic characteristics of women seeking antenatal care in Iringa region hospital, Southern Tanzania in July to September 2009

\begin{tabular}{|l|l|l|l|}
\hline Variables & & $\mathbf{N}$ & \% \\
\hline \multirow{4}{*}{ Age } & & & \\
\cline { 2 - 4 } & $14-19$ & 37 & 16.7 \\
\cline { 2 - 4 } & $20-24$ & 86 & 38.7 \\
\cline { 2 - 4 } & $25-29$ & 57 & 25.7 \\
\cline { 2 - 4 } & $30-34$ & 23 & 10.4 \\
\hline
\end{tabular}




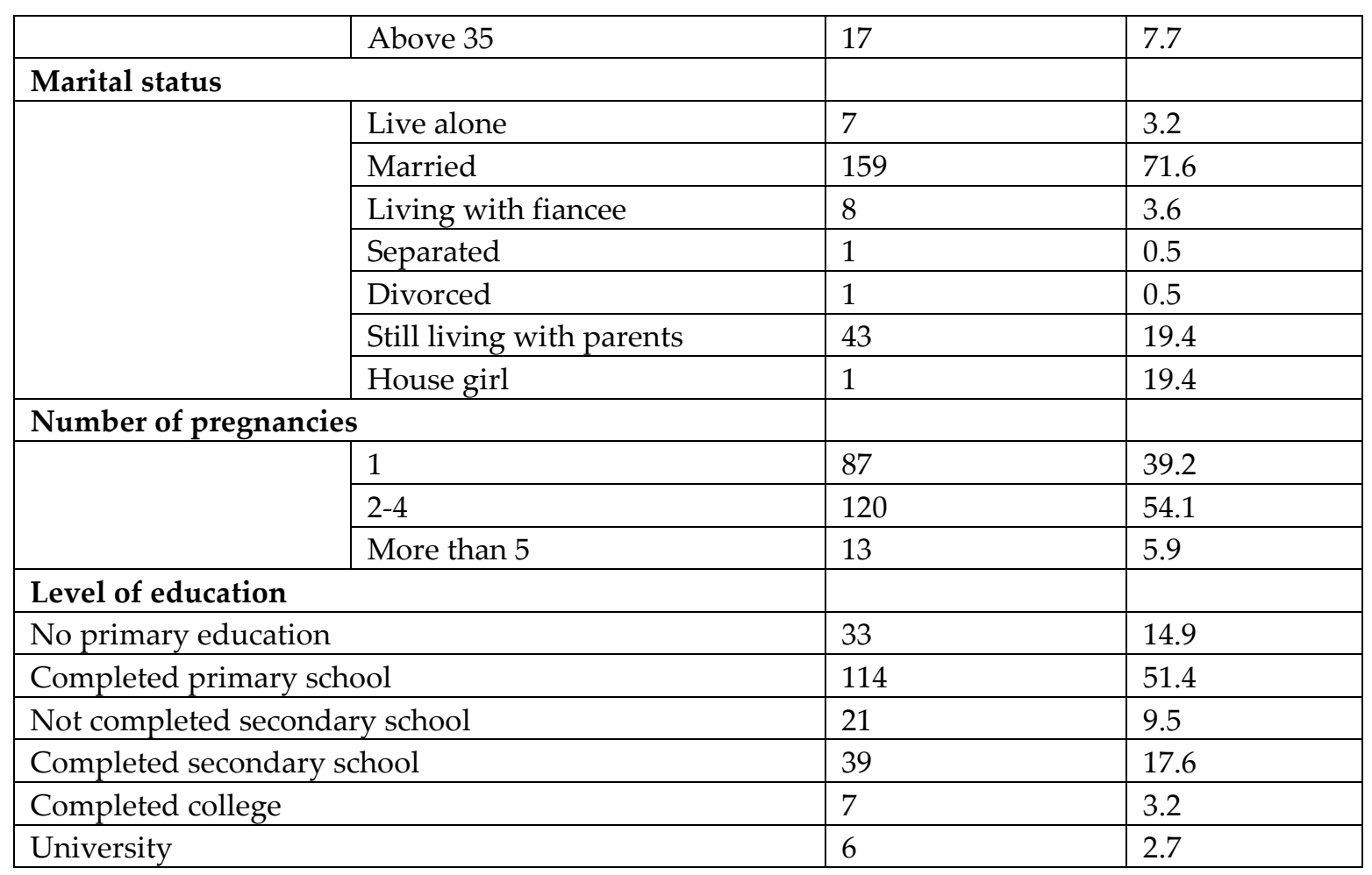

\section{Knowledge of malaria and use of bed nets}

All participants reported they had heard of malaria, and $178(80.2 \%)$ knew that the disease is transmitted by mosquitoes (Table 2). The major method of malaria prevention used by respondents was sleeping under bed nets (64\%). The vast majority of women had been diagnosed with malaria during the past year, with most diagnoses made by a physician (Table 2). In total, $173(77.9 \%)$ of the pregnant women reported to own a mosquito nets (ITNs and untreated bed nets) and $150(68 \%)$ reported to sleep always under a bed net (Table 2). Of those having mosquito nets, 170 (98.3\%) owned between 14 mosquito nets in their houses. The majority of those who did not own a mosquito bed nets, reported the costs to be the main barrier for not owning a net (Table 2). When asked about the reasons for not sleeping under a net, $45.6 \%$ of women stated not having a net (Table 2).

Table 2: Knowledge of malaria and use of mosquito nets among antenatal clinic attendees Table 2: Knowledge of malaria and use of mosquito nets among antenatal clinic attendees

\begin{tabular}{llll}
\hline Variable & Response & $\mathbf{N}^{*}$ & \% \\
\hline Causes of malaria (unprompted) & Mosquito & 178 & 80.2 \\
& Dirty environment & 2 & 0.9 \\
& Standing water & 4 & 1.8 \\
& Mosquito and standing water & 3 & 1.4 \\
& Mosquito and dirty & 1 & 0.5 \\
& environment & & \\
Do you know ways to prevent & I don't know & 22 & 9.9 \\
& Yes & 204 & 91.9
\end{tabular}


malaria?

$\begin{array}{llll}\text { What are the ways to prevent } & \text { No } & 15 & 6.8 \\ \text { Mosquito net } & 142 & 64.0\end{array}$

malaria?

\begin{tabular}{|c|c|c|}
\hline Environmental cleanliness & 3 & 1.4 \\
\hline Antmalarial drugs & 7 & 3.2 \\
\hline $\begin{array}{l}\text { Mosquito net and } \\
\text { environmental cleanliness }\end{array}$ & 12 & 5.4 \\
\hline $\begin{array}{l}\text { Mosquito net and } \\
\text { antmalarials }\end{array}$ & 17 & 7.7 \\
\hline $\begin{array}{l}\text { Mosquito net, antmalarial } \\
\text { and } \\
\text { cleanliness }\end{array}$ & 19 & 8.6 \\
\hline I don't know & 19 & 8.6 \\
\hline Yes & 187 & 84.2 \\
\hline No & 32 & 14.4 \\
\hline Self diagnosis & 8 & 3.6 \\
\hline Physician & 181 & 81.5 \\
\hline Yes & 173 & 77.9 \\
\hline No & 46 & 20.7 \\
\hline 1 & 85 & 49.1 \\
\hline
\end{tabular}

Number of mosquito nets owned 1

Malaria diagnosis in the past Yes

Who diagnosed your malaria?

Self diagnosis

14.4

Do you own mosquito nets?

in the

house hold $(n=173)$

\begin{tabular}{llll} 
& $2-4$ & 82 & 47.4 \\
& $4-5$ & 2 & 1.2 \\
$\begin{array}{l}\text { Do you always sleep } \quad \text { under } \\
\text { mosquito nets }\end{array}$ & yes & 4 & 2.3 \\
& & 150 & 67.6 \\
Reasons for why not to sleep under & no & & \\
bed nets always (n=68) & & 68 & 30.6 \\
& My bed net was torn & 31 & 45.6 \\
& I get tired & 4 & 5.9 \\
& No mosquito & 7 & 10.3 \\
& I forget & 8 & 11.8 \\
Reason for not owning a mosquito & I dislike & 5 & 7.4 \\
net (unprompted) $\mathbf{n}=\mathbf{4 6}$ & Cost (very expensive) & 3 & 4.4 \\
& It was damaged & 34 & 73.9 \\
& I don't like & 3 & 6.5 \\
& No mosquito in my area & 8 & 2.2 \\
& Not interested in bed nets & 1 & 17.4 \\
\hline
\end{tabular}

*For some variables, responses were not available in all cases. 


\section{Attitudes about malaria}

Attitudes about malaria and bed net use are depicted in Table 3. The vast majorities of participants were worried about getting malaria, stated that it was a serious disease, and believed that it is important and beneficial to sleep under a mosquito net every night. About half reported that they would prefer to use a net they had bought by themselves, compared to one distributed for free (Table 3). Only 18.5\% reported being unable to afford buying a mosquito net if they would not receive one for free.

\section{Factors associated with mosquito net ownership}

Several factors such as gravidity $(P<0.001)$, knowledge on the cause of malaria $(P<0.001)$ and knowledge to prevent malaria $(P<0.001)$, fearing to get malaria $(P<0.001)$ and marital status were associated with bednets ownership among pregnant women. Age $(P=0.269)$ and education level $(P=0.152)$ were not associated with mosquito net ownership. In bivariate analysis, multigravidae (2-4 pregnancies) (OR 2.1, 95\%CI 1.2-4.8) and married women (OR, 1.9, 95\%CI, 1.2-5.2) were more likely to own a net compared to primigravidae and single women. Furthermore, factors such as gravidity (multigravidae, $2-4$ pregnancies) $(P<0.001)$, education levels $(P<0.002)$ and fearing to get malaria $(\mathrm{P}<0.001)$ were associated with the behaviour of sleeping under mosquito nets among net owners.

Table 3: Attitudes about malaria and bed net use among women seeking antenatal care

\begin{tabular}{|c|c|c|}
\hline & $\mathbf{N}$ & $\%$ \\
\hline \multicolumn{3}{|l|}{ In your opinion, do you think that most people where you live } \\
\hline Are worried/concerned about getting malaria & $190 / 204$ & 93.1 \\
\hline $\begin{array}{l}\text { Believe that using mosquito nets is the best way for prevention } \\
\text { against malaria }\end{array}$ & $210 / 216$ & 97.2 \\
\hline $\begin{array}{l}\text { Prefer using a mosquito net that they have brought rather than one } \\
\text { given to them for free }\end{array}$ & $117 / 160$ & 73.1 \\
\hline $\begin{array}{l}\text { In your own opinion, do you believe } \\
\text { that are you worried of getting malaria }\end{array}$ & $144 / 219$ & 65.7 \\
\hline $\begin{array}{l}\text { it is important and beneficial to sleep under a mosquito nets every } \\
\text { night }\end{array}$ & $217 / 219$ & 99 \\
\hline $\begin{array}{l}\text { you would prefer using a mosquito net you have bought better than } \\
\text { one given to you for free }\end{array}$ & $80 / 148$ & 54.1 \\
\hline $\begin{array}{l}\text { that you wouldn't afford to buy a mosquito if you didn't receive one } \\
\text { for free }\end{array}$ & $38 / 206$ & 18.5 \\
\hline $\begin{array}{l}\text { it is useless to use a mosquito net because you can suffer from } \\
\text { malaria anyway }\end{array}$ & $10 / 206$ & 4.9 \\
\hline that obtaining mosquito net in the community where you live is easy & $114 / 212$ & 53.8 \\
\hline that malaria is a serious disease & $217 / 220$ & 98.6 \\
\hline $\begin{array}{l}\text { that it is more difficult to use a mosquito net than taking drugs when } \\
\text { you suffer from malaria }\end{array}$ & $17 / 211$ & 8.1 \\
\hline that children suffer from malaria more than adult do & $159 / 163$ & 97.5 \\
\hline it's difficult to use a mosquito net & $7 / 212$ & 3.3 \\
\hline
\end{tabular}




\section{Discussion}

The current death rates from malaria in Africa are undoubtedly decreasing because of improvement in health services, individual and community increased use of malaria intervention tools such as ITNs. Currently, malaria endemic countries are optimistic to eliminate the disease in the near future, however, the control of malaria requires good knowledge and awareness of appropriate preventive measures among the general public to ensure positive health behavior changes and health seeking habits (Minja et al., 2001; Panter-Brick et al., 2006).

Uses of ITNs among pregnant women have been found to be increasing in different areas of Africa. In community surveys of six sub-Saharan countries, use of ITNs by women of reproductive age varied from $32 \%$ to $69 \%$ (Baume et al., 2007). In another survey in Burkina Faso where pregnant women were interviewed in ANC clinics and Delivery Units, 58\% reported owning an ITN (Sirima et al., 2006). Majority of the pregnant women in the present study reported to own bed nets and about 2/3 reported always to sleep under a bed net. This observation gives a promising future to attain the Roll Back Malaria (RBM) partnership goal of $80 \%$ mosquito net coverage in pregnant women. At the time of this study, bed nets were distributed freely to mothers of underfives, as part of the Tanzania government strategies to control malaria. This could partly explain the high level of mosquito net ownership in multigravidae women. Pregnant mothers were also receiving subsidized ITNs under voucher schemes when they attended antenatal clinics. However, not all pregnant women who attend ANC can afford to pay for the subsidized nets and the system have been critised to favour the better off (Matovu et al., 2009). Alternative sources of ITNs for pregnant women are private sectors such as retail shops (Matovu et al., 2009). This is clearly demonstrated in our study, where more than $50 \%$ of the pregnant women reported to obtain their nets from the private sector. Accessibility of ITNs from retail shops is easier for the pregnant women in the present study area; however, the high cost could be an obstacle (Osero et al., 2006; Noor et al., 2006; Matovu et al., 2009; Mazigo et al., 2010). Lack of affordability was reported as an important barrier to ITNs ownership (Pulford et al., 2011). Previous studies have found that free distribution of ITNs has resulted in substantial increase in net coverage compared to subsidize (Noor et al., 2006; Maxwell et al., 2006). Thus, mass and free distribution of ITNs might be necessary to achieve the targets of Roll Back Malaria (RBM), however, this will not guarantee the use of the interventions (PanterBrick et al., 2006). To increase the uptake and use of the malaria intervention by the targeted group or community, the designed interventions should be build on the existing practices, culture and the community must be fully engaged (Panter-Brick et al., 2006).

The overall understanding of malaria transmission and effective means to prevent transmission were good in the present study population. About $65 \%$ of the women reported being worried about contracting malaria and reported positive attitudes towards using bed nets to prevent malaria. Factors such as gravidity, 
knowledge on the cause and preventive measures against malaria, fearing to contract malaria and being married were strongly associated with net ownership. However, age and education level of pregnant women were not associated with bed net ownership and use. This stands in contrast with other studies in Africa, which have reported that women with higher education were more likely to own and use bed nets (Osero et al., 2006; Noor et al., 2007; Mazigo et al., 2010).

Our study is subject to limitations. We acknowledge the fact that the ANC-based study population may not be representative for the general population. Furthermore, the study sample came from a single clinic and all the information used in the present study relied on the individual reporting on the ownership and use of ITNs.

In conclusion, acceptability of mosquito nets, and net ownership and use are promising in the study population. To achieve maximum ITNs distribution and coverage among risk groups such as pregnant women, there is a need to integrate public and private sector.

\section{Competing interests}

None

\section{References}

Baume, C.A. \& Marin, M.C. (2007) Intra-household mosquito nets use in Ethiopia, Ghana, Mali, Nigeria, Senegal and Zambia: Are nets being used? Who in the household uses them? American Journal of Tropical Medicine and Hygiene 77, 963971.

D’Alessandro, U., Langerock, P., Bennett, S., Francis, N., Cham, K. \& Greenwood, B.M.(1996) The impact of a national impregnated bed net programme on the outcome of pregnancy in primigravidae in The Gambia. Transactions of the Royal Society of the Tropical Medicine and Hygiene 90, 487-492.

Matovu, F., Goodman, C., Wiseman, V. \& Mwengee, W. (2009) How equitable is bed net ownership and utilisation in Tanzania? A practical application of the principles of horizontal and vertical equity. Malaria Journal 8:109.

Minja, H., Schellenberg, J.A., Mukasa, O., Nathan, R., Abdullah, S., Mponda, H., Tanner, M., Langeler, C. \& Obrist, B. (2001). Introducing insecticide-treated nets in the Kilombero Valley, Tanzania: the relevance of local knowledge and practice for an Information, Education and Communication (IEC) campaign. Tropical Medicine and International Health, 6, 614-623.

Maxwell, C.A., Rwegoshora, R.T., Magesa, S.M. \& Curtis, C.F. (2006) Comparison of coverage with insecticides-treated nets in a Tanzanian town and villages where nets and insecticides are either marketed or provided free of charge. Malaria Journal 5:44

Mazigo, H.D., Obasy, E, Mauka, W., Manyiri, P., Zinga, M., Kweka, E.J., Mnyone, L.L. \& Heukelbach, J. (2010) Knowledge, attitudes and practices about malaria and its 
control in rural northwest Tanzania. Malaria Research and Treatment doi10.4061/2010/794261.

Noor, A.M., Amin, A.A., Akhwale, W.S. \& Snow, R.W. (2007) Increasing coverage and decreasing inequity in insecticides-treated bed nets use rural Kenyan children. PLos Medicine 4:8; e255.

Noor, A.M., Omumbo, J.A., Amin, A.A., Zurovac, D. \& Snow, R.W. (2006) Wealth, mother's education and physical access as determinants of retail sector net use in rural Kenya. Malaria Journal 5:5.

Opiyo, P., Mukabana, R., Kiche, I., Mathenge, E., Killen, G.F. \& Fillinger, U.(2007). An explanatory study of community factors relevant for participatory in malaria control on Rusinga Island, western Kenya. Malaria Journal 6: 48.

Osero, J.S., Otieno, M.F. \& Orago, A.S. (2006) Mother's knowledge on malaria and vectors management strategies in Nyamira District, Kenya. East African Medical Journal 83, 507-514.

Panter-Brick, C., Clarke, S.E., Lomas, H., Pinder, M. \& Lindsay S.W.(2006). Culturally compelling strategies for behaviour change a social ecology model and case study in malaria prevention. Social Science and Medicine 62, 2810-2825.

Pulford, J., Hetzel, MW., Bryant, M., Siba, P.M. \& Mueller, I. (2011) Reported reasons for not using a mosquito net when one is available: a review of the published literature. Malaria Journal 10:83.

Roll Back Malaria (2002) Children and Malaria. Roll Back Malaria Fact Sheets. Geneva; World Health Organization, 2002.

Roll Back Malaria (2002) Malaria in Africa. Roll Back Malaria Fact Sheets. Geneva; World Health Organization, 2002.

Roll Back Malaria(2002) Malaria in Pregnancy. Roll Back Malaria Fact Sheets. Geneva; World Health Organization, 2002.

Roll Back Malaria, World Health Organization, United Nations Children Fund.(2005). World Malaria Report, 2005, Geneva: World Health Organization, 2005.

Sirima, S.B., Cotte, A.H. \& Konate, A. (2006) Malaria prevention during pregnancy: assessing the disease burden one year after implementing a program of intermittent strategies in Koupela District, Burkina Faso. American Journal of Tropical Medicine and Hygiene 77, 205-211.

Teklehaimonot, A., Sachs, J.D. \& Curtis, C. (2007) Malaria Control needs mass distribution of insecticidal bed nets. The Lancet 369, 2143-2146.

ter Kuile, F.O., Terlouw, D.J., Phillips-Howard, P.A., Hawley, W.A., Friedman, J.F., Kariuki, S.K., Shi, Y.P., Kolczak, M.S., Lal, A.A., Vulule, J.M. \& Nahlen, B.L.(2003) Reduction of malaria during pregnancy by permehthrin-treated bed nets in an area of intense perennial malaria transmission in western Kenya. American Journal of Tropical Medicine and Hygiene 68 (Suppl 4), 50-60.

WHO/UNICEF. (2003). The Africa Malaria Report for year 2003.pp 9-29

Wiseman, V., Scott, A., McElroy, B., Conteh, L. \& Stevens, W. (2007) Determinants of Bed Nets Use in the Gambia: Implications for Malaria Control. American Journal of Tropical Medicine and Hygiene 76, 5:830-836. 